\title{
Implantable cardioverter defibrillators - the past, present and future
}

\author{
Ganesh Kumar K. Ammannaya
}

AATS Visiting Fellow, NYU Langone Health, Department of Cardiothoracic Surgery, NY, USA

Submitted: 7 May 2020

Accepted: 31 May 2020

Arch Med Sci Atheroscler Dis 2020; 5: e163-e170

DOI: https://doi.org/10.5114/amsad.2020.97103

Copyright $\odot 2020$ Termedia \& Banach

\author{
Corresponding author: \\ Dr. Ganesh Kumar K. \\ Ammannaya, MS, MCH, \\ FEBCTS, FACS, FICS, FACC \\ AATS Visiting Fellow \\ NYU Langone Health \\ NY, USA \\ E-mail: doc.ammannaya@ \\ gmail.com
}

\begin{abstract}
Since their formal introduction in 1980, implantable cardioverter defibrillators (ICDs) have undergone innumerable design modifications through several generations. They are indispensable today in successfully managing fatal ventricular arrhythmias. Their role in averting sudden cardiac death is recognized beyond doubt. Their applications and indications have continuously expanded over the last two decades. This article reviews the salient features in the evolution of ICDs, their current indications, recent advances and future directions. With more advanced detection algorithms, the potential integration with leadless pacing, and the possibility to serve as a remote monitoring device to recognize atrial fibrillation, acute ischemia, or electrolyte imbalance, the application of ICDs is rapidly evolving.
\end{abstract}

Key words: implantable cardioverter defibrillators, sudden cardiac death, ventricular arrhythmias.

\section{Introduction}

Implantable cardioverter defibrillators (ICDs) are electronic devices that are intricately designed with the purpose of detecting as well as terminating cardiac arrhythmias. With added capabilities of demand pacing, their applications are two-fold - emergency defibrillation and backup pacing. Initially looked upon as a remote choice for patients with sustained ventricular tachycardia (VT), their effectiveness is unquestioned today [1-3]. Over 200,000 ICDs are implanted worldwide every year, with rapidly expanding applications in modern day medicine [4].

\section{Prologue to the birth of ICDs}

Beck described open chest defibrillation of the human heart for the first time in 1947 [3, 5]. A decade later, Kouwenhoven showed that it was possible to defibrillate the heart through a closed chest. The weight of the first external defibrillator so employed was a massive $120 \mathrm{~kg}$ and delivering an alternating current $(\mathrm{AC})$ of $500 \mathrm{~V}$, thus limiting its practical application $[3,6]$. Lown, in 1962, developed a direct current (DC) defibrillator, which consisted of a capacitor for storing energy, a battery and a transformer to overcome the high incidence of cardiac arrhythmias and cardiac damage that occurred with AC current [3, 7]. It was not until 1970 that the use of an implantable defibrillator was first demonstrated by John Schuder, at the University of Missouri in 1970, on a canine model [2, 3, 7-9]. 


\section{Conception of ICDs in humans and the early design}

1980 was the landmark year that saw the first successful implantation of ICD in a human by Michel Mirowski $[3,10]$. The twist of events leading to this feat was rather interesting. It was the sudden and unfortunate death of his colleague, Dr. Harry Heller in 1966, due to VT, that led Mirowski to conceive the idea of an ICD. In 1968, Mirowski was hired by the Sinai Hospital in Baltimore as the director of the coronary care unit, with ample time for research. The biomedical engineering division and animal laboratory of the hospital aided research $[3,11]$. The early fruit was the successful testing of the first crude prototype on a dog in $\mathrm{Au}$ gust 1969 [3, 9, 10, 12]. Eventually, Mirowski and Mower garnered support from a major pacemaker company in 1970 to further develop the ICD, only to be abandoned 2 years later, as the company saw no market for the device.

In 1972, Mirowski came in touch with Stephen Heilman, a physician-cum-engineer who founded Medrad, a medical equipment company. Heilman, who was highly impressed by the novel idea of ICD, put his engineers at Mirowski's disposal. This partnership yielded the first ICD prototype small enough to be implanted in a dog in 1975. A film of the first successful defibrillation of a dog implanted with the prototype ICD was released and this famous footage propelled ICD into the limelight overnight $[3,11]$.

Further refinements in the prototype were made to make it suitable for human implantation. Food and Drug Administration (FDA) approval was eventually received. Ably supported by his colleagues Myron Weisfeldt (cardiac surgeon) and Philip Reed (electro physiologist), at Johns Hopkins Hospital, the first human implant of an ICD was performed successfully in February 1980 [3, $9,10,13]$. A 57-year-old woman with severe coronary artery disease with recurrent VA refractory to all standard antiarrhythmic drugs was the first to receive the device [3]. The ICD could successfully terminate a ventricular flutter induced during an electrophysiological study 2 weeks later. After five months, the patient had been without VA. The second patient was a 16-year-old boy with refractory VA. Five episodes of hypotensive VT were cardioverted successfully by the device 2 weeks after implantation $[3,10]$.

\section{Early design}

The devices implanted in the 1980s were designed merely to recognize and terminate ventricular fibrillation (VF) by delivering a high-energy shock [10]. These early devices lacked the ability to detect unstable VT that could degenerate into
VF. Moreover, as these devices were not programmable, separate pacemakers were needed to allow backup bradycardia pacing, leading to lethal interactions [10, 12, 14]. It weighed $250 \mathrm{~g}$ and had a volume of $145 \mathrm{ml}$. An open thoracotomy was required for implantation of the epicardial patch electrodes and the device was implanted in the abdomen. The electrodes were made of titanium and silicone rubber. One electrode was placed epicardially on the cardiac apex while a second electrode sat in the right ventricular apex. The battery was made of titanium and was hermetically sealed to prevent moisture from reaching the capacitor and other circuitry. Within $15 \mathrm{~s}$ of induced VF, the device delivered a 30 joule shock to convert the rhythm. The life of the lithium battery was projected to be 3 years or 100 shocks [3]. In the following years, numerous advances in ICD design were made. Mirowski and Mower pioneered several of these early refinements $[3,10,15]$.

\section{Evolution to the present day form - through the generations}

As a major improvement over the early devices, the second generation ICDs were designed to detect ventricular arrhythmias (VA) using a probability density parameter based on the concept that, unlike sinus rhythm, ventricular fibrillation did not maintain an isoelectric baseline. This enabled bradycardia pacing ability and they were minimally programmable. This ended the need for separate pacemakers. The second generation ICDs could do away with thoracotomy by the introduction of transvenous leads in 1988, which enabled the implantation procedure to be performed in an electrophysiology laboratory rather than open surgery [16]. Furthermore, these devices possessed limited telemetry function to test battery strength, for which an external monitoring device was needed. Cylindrical aluminium electrolytic capacitors and silver vanadium pentoxide batteries of the first generation ICDs were replaced by lithium-silver vanadium manganese oxide batteries, which resulted in longer life of ICDs [14].

The first third generation ICDs were introduced in the early 1990s. Anti-tachycardia pacing (ATP), low energy shocks for terminating VTs, high level of programmability and telemetry functions were the key upgrades [17]. To improve specificity in discriminating between VT or supraventricular tachycardia, various algorithms were developed. ICDs could be programmed into three different cycle length-related zones and the discriminative detection algorithms programmable in the two lowest zones. The highest programmable zone is meant for detection of fast VT or VF without any further discrimination to avoid unnecessary delay of delivery of therapy. Additionally, improvements 
were made in lead construction. The coaxial lead design of the first and second generation ICDs was replaced with the multi-lumen lead design in third generation ICDs. The coaxial lead had a layered design comprising a tip conductor, ring conductor, defibrillation conductor and an insulation layer between each conductor. The multi-lumen lead construction is based on parallel running conductors through a single insulating body. The key advantage of multi-lumen over coaxial leads is the fact that more conductors would fit into overall smaller leads. The tip and ring conductors are used for pacing and sensing, a defibrillation conductor for the coil located in the right ventricle and a defibrillation conductor for the coil located in the superior vena cava. The insulating body contains extra lumens to increase the lead's resistance to compression forces [18].

ICDs of today or the fourth generation ICDs have progressively become smaller and more sophisticated. They weigh not more than 80-90 g and have a volume of $30 \mathrm{ml}$, measuring less than a centimeter in thickness. Newer lithium silver vanadium batteries now last up to 9 years [19]. All modern ICDs carry the ability of overdrive pacing, i.e. ATP, which can often terminate VTs without resorting to shock therapy. In addition, ICDs are also available with biventricular pacing (cardiac resynchronization therapy) to improve symptoms in patients with advanced cardiac failure [2].

\section{Indications for ICDs}

Indications for ICDs can be broadly categorized as primary prophylaxis and secondary prophylaxis against SCD. Multiple randomized clinical trials have shown ICDs to be clearly superior to antiarrhythmic drugs in patients with a history of life-threatening VT and VF and therefore the indications for secondary prophylaxis are well supported by clinical evidence. However, indications for primary prophylaxis, representing most of the ICD implants, have comparatively less well established evidence, as the measurable quantitative benefit is smaller [1].

Wilcox et al. [20] studied the clinical effectiveness of cardiac resynchronization (CRT) and ICD therapy among 8936 outpatients with heart failure and reduced ejection fraction (EF) $\leq 35 \%$ and found a substantially reduced 24-month mortality in eligible men and women with heart failure and reduced EF. Pokorney et al. [21] investigated the relationship between primary prophylaxis of ICDs and mortality in $852 \mathrm{racial} / \mathrm{ethnic}$ minority patients (non-white race or Hispanic ethnicity) and 2070 white non-Hispanic patients. Minority ICD patients showed a lower adjusted 3-year mortality rate $(44.9 \%)$ as compared to their non-ICD counterparts (54.3\%). Similarly, white non-Hispanic
ICD patients showed a lower adjusted 3-year mortality rate $(47.8 \%)$ in comparison to their non-ICD counterparts (57.3\%). No significant relationship between race/ethnicity and lower mortality risk with ICD was found and the results concur with the use of a similar approach in selecting ICD patients, irrespective of race or ethnicity.

Several randomized control trials have shaped the present day indications for ICDs. Some of the major trials include MADIT I \& II, CAT, MADIT-CRT, PRESERVE EF and PROTECT-ICD. The MADIT-I study showed that ICD saves lives in high-risk patients with coronary heart disease. The MADIT-II study showed that prophylactic ICD therapy was associated with significantly improved survival in patients with ischemic cardiomyopathy, as defined by documented coronary heart disease and advanced left ventricular dysfunction, without requiring screening for ventricular arrhythmias or inducibility by electrophysiologic testing. Together, these studies, as well as the results from several other randomized ICD trials, indicate that ICD therapy is indicated in coronary patients who meet MADIT-I or MADIT-II eligibility criteria, who are not excluded by major non-cardiac comorbidity [22]. The CAT trial did not provide evidence in favor of prophylactic ICD implantation in patients with DCM of recent onset and impaired left ventricular ejection fraction [23]. Patients with New York Heart Association class I or II heart failure, ejection fraction $\leq 30 \%$ and left bundle branch block (LBBB) derive substantial clinical benefit from CRT-D in the form of a reduction in heart failure progression and a reduction in the risk of ventricular tachyarrhythmias. Cardiac resynchronization therapy (CRT) thus evolved as a Class I treatment indication with Level of Evidence $A$, in patients with mild heart failure, depressed left ventricular ejection fraction, and wide QRS [24]. Current classification of SCD vs. NSCD fails to identify VTA events at the time of death in a significant proportion of patients. Multivariate analysis showed that factors independently associated with VTA mortality included VT/VF $>72 \mathrm{~h}$ prior to the mortality event, hospitalization for heart failure, and a history of hypertension. SCD annual incidence is $0.6-1 \%$ in post-MI patients with left ventricular ejection fraction (LVEF) $\geq 40 \%$. No recommendations for ICD exist in this population. The two-step approach of the PRESERVE EF study detects a subpopulation of post-myocardial infarct (post-MI) patients with preserved LVEF at risk for MAEs that can be effectively addressed with an ICD [25]. The Programmed Ventricular Stimulation to Risk Stratify for Early Cardioverter-Defibrillator Implantation to Prevent Tachyarrhythmias following Acute Myocardial Infarction (PROTECT-ICD) trial is an ongoing trial targeting the prevention of 
sudden cardiac death in patients who have at least moderately reduced cardiac function following a MI. The primary objective of the trial is to assess whether electrophysiological study to guide prophylactic implantation of an ICD early following MI (first 40 days) will lead to a significant reduction in sudden cardiac death and non-fatal arrhythmia. The secondary objective is to assess the utility of cardiac MRI (CMR) in assessing early myocardial characteristics, and its predictive value for both inducible ventricular tachycardia (VT) at EPS and SCD/non-fatal arrhythmia at follow-up [26].

The complete list of indications for ICDs are listed in Table I as per the 2017 American College of Cardiology, American Heart Association and Heart Rhythm Society (ACC/AHA/HRS) guidelines [1].

\section{Wearable cardioverter defibrillators (WCDs), subcutaneous implantable cardioverter defibrillators (S-ICDs) and future perspectives}

Introduced in 1998 [27], at the University Hospital Magdeburg, Germany, wearable cardioverter defibrillators (WCDs) were approved by the US FDA in 2001. WCDs are now frequently recommended for patients considered to be at high arrhythmic risk with potentially reversible underlying pathology or who are awaiting an ICD implantation to bridge a period of high risk of SCD [28].

A well-fitting electrode belt is necessary to ensure reliable arrhythmia detection. The harness comprises four sensing electrodes (anterior, posterior, right and left) that generate a two-lead filtered ECG. The arrhythmia detection algorithm combines heart rate data and morphology analysis. The patient's consciousness is evaluated by assessing the response to the escalating alerts. Response buttons are present on either side of the monitor. The patient is required to press and hold both buttons simultaneously for therapy to be withheld. If the patient does not press or release them, the escalating warning system continues until an audible message to stand clear is emitted for bystanders. The patient interaction feature contributes to optimization of the sensitivity and specificity of the algorithm [29]. Patient compliance is mandatory for appropriate performance of the WCD. Careful training and instruction prior to WCD wearing are very important. Appropriate patient training for the correct use of the WCD is provided by the technical personnel of the manufacturer and includes instructions on battery charging and data transfer to the network server. After the initial training, which may last an hour or more, the technical personnel contact the patient within the next few days to avoid potential problems of device use. Patients or their relatives can contact the $24 / 7$ hotline for queries or in case of problems.
The ACC/AHA/HRS Guidelines 2017 included WCDs. The indications for the use of WCDs are listed in Table II [1]. WCDs have been successful not only in terminating arrhythmic events, but also in preventing unnecessary ICD implantation and its associated morbidity.

An entirely subcutaneous ICD (S-ICD) is a significant step forward towards a less invasive approach. As transvenous ICDs still carry considerable morbidity ( $1.5 \%$ major complications) [30] and other procedural risks such as endocarditis, vessel occlusion, lead dislodgment, valvular dysfunction and intrinsic lead defects/failure and infections, S-ICDs offer an attractive alternative to prevent these shortcomings of transvenous leads. The S-ICDs received the CE mark and US FDA approval in June 2009 and September 2012, respectively [31].

S-ICDs are equipped with extrathoracic, subcutaneous electrodes, thus completely avoiding transvenous leads and related morbidity. The defibrillation coil ( $8 \mathrm{~cm}$ in length) lies directly between two sensing electrodes, while the S-ICD generator acts as the 3rd electrode, used for sensing and defibrillation. The pulse generator serves as a mandatory component of the defibrillation pathway and as an optional electrode for sensing [31]. The newest generation of S-ICD incorporates wireless connectivity to secure servers to deliver health-related information to remote caregivers. S-ICDs require a careful selection process to avoid both oversensing (T-wave) and undersensing (R-wave) leading to catastrophic consequences. One of their potential limitations, however, is in cases where a higher defibrillation threshold is to be expected (e.g. HCM) [32].

The 2017ACC/AHA/SRS guidelines included the use of S-ICD. The current indications for the use of S-ICD are listed in Table III [1].

\section{Future perspectives}

ICDs will continue to evolve, become smaller, equipped with more advanced detection algorithms to treat life-threatening arrhythmias. In the future, the potential integration of S-ICDs with leadless pacing may make this therapy suitable for a larger target population at high risk of SCD. Moreover, if the leadless pacemaker is epicardial, left atrial and left ventricular pacing can be introduced and linked to S-ICD; alternatively application of a left ventricular seed would permit resynchronization [33]. S-ICDs may also serve as a remote monitoring device to recognize atrial fibrillation, acute ischemia, or electrolyte imbalance [34].

New ICD and CRT-D devices such as the new Gallant (Abbott Inc., USA) have received the CE Mark recently in February 2020. This new Gallant 
Table I. Indications for ICDs (ACC/AHA/HRS Guidelines 2017) [1]

Indications [1]

1. Patients with IHD surviving SCA due to VT/VF or experiencing hemodynamically unstable VT or stable sustained VT not due to reversible causes, if meaningful expected survival $>1$ year [1]

2. Patients with IHD with unexplained syncope and inducible sustained monomorphic VT on EPS, if meaningful expected survival > 1 year [1]

3. In patients with LVEF $\leq 35 \%$ due to IHD who are $\geq 40$ days' post-MI and $\geq 90$ days post-revascularization with NYHA class II/III HF despite GDMT, if meaningful expected survival $>1$ year [1]

4. In patients with LVEF $\leq 30 \%$ due to IHD who are $\geq 40$ days' post-MI and $\geq 90$ days post-revascularization with NYHA class I HF despite GDMT, if meaningful expected survival $>1$ year [1]

5. In patients with NSVT due to prior MI, LVEF $\leq 40 \%$ and inducible sustained VT/VF at EPS, if meaningful expected survival $>1$ year [1]

6. In patients with NICM surviving SCA due to VT/VF or experiencing hemodynamically unstable VT or stable sustained VT not due to reversible causes, if meaningful expected survival $>1$ year [1]

7. In patients with NICM, NYHA class II-III HF with LVEF $\leq 35 \%$, despite GDMT, if meaningful expected survival $>1$ year [1]

8. In patients with arrhythmogenic right ventricular cardiomyopathy and increased risk of SCD (resuscitated SCA, sustained VT, significant ventricular dysfunction with RVEF or LVEF $\leq 35 \%$ ), if meaningful expected survival $>1$ year [1]

9. In patients with HCM surviving an SCA due to VT/ VF, or having spontaneous sustained VT causing syncope or hemodynamic compromise, if meaningful expected survival $>1$ year [1]

10. In patients with cardiac sarcoidosis with sustained VT or survivors of SCA or have an LVEF $\leq 35 \%$, if meaningful expected survival $>1$ year [1]

11. In patients with neuromuscular disorders, ICDs are recommended for the same indications as for patients with NICM if meaningful expected survival > 1 year [1]

12. In patients with a cardiac channelopathy and SCA, if meaningful expected survival > 1 year [1]

13. In patients with catecholaminergic polymorphic ventricular tachycardia and recurrent sustained VT or syncope, while receiving adequate or maximally tolerated $\beta$-blocker, treatment intensification with combination medication therapy (e.g. $\beta$-blocker, flecainide), left cardiac sympathetic denervation [1]

14. In patients with Brugada syndrome with spontaneous type 1 Brugada electrocardiographic pattern and cardiac arrest, sustained VA or a recent history of syncope presumed due to VA, if meaningful expected survival $>1$ year [1]

15. In patients with early repolarization pattern on ECG and cardiac arrest or sustained VA, if meaningful expected survival > 1 year [1]

16. In patients with short QT syndrome with a cardiac arrest or sustained VA, if meaningful expected survival $>1$ year [1]

17. In patients resuscitated from SCA due to idiopathic polymorphic VT or VF if meaningful expected survival $>1$ year [1]

18. In patients with adult congenital heart disease and hemodynamically unstable VT, if meaningful expected survival $>1$ year [1]

19. In patients with adult congenital heart disease with SCA due to VT or $V F$ in the absence of reversible causes, if meaningful expected survival $>1$ year $[1]$
Class [1]

Class I

(The treatment is useful, its benefit greatly outweighs the risk, and it should be administered) [1]

Class I

(The treatment is useful, its benefit greatly outweighs the risk, and it should be administered) [1]

Class I

(The treatment is useful, its benefit greatly outweighs the risk, and it should be administered) [1]

Class I

(The treatment is useful, its benefit greatly outweighs the risk, and it should be administered) [1]

Class I

(The treatment is useful, its benefit greatly outweighs the risk, and it should be administered) [1] 
Table I. Cont.

Indications [1]

Class [1]

1. Patients with NYHA class IV symptoms who are candidates for cardiac transplantation or LVAD, if meaningful expected survival > 1 year [1]

2. In patients with NICM experiencing syncope presumed to be due to VA and who do not meet indications for a primary prevention ICD, if meaningful expected survival $>1$ year [1]

3. In patients with NICM due to a Lamin A/C mutation with 2 or more risk factors (NSVT, LVEF $\leq 45 \%$, non-missense mutation, and male sex), if meaningful expected survival $>1$ year [1]

4. In patients with arrhythmogenic right ventricular cardiomyopathy and syncope due to VA, if meaningful expected survival $>1$ year [1]

5. In patients with HOCM and $\geq 1$ of the following risk factors, if meaningful expected survival $>1$ year: a. Maximum LV wall thickness $\geq 30 \mathrm{~mm}$. b. SCD in $\geq 1$ first-degree relatives caused by HCM. c. $\geq 1$ episodes of unexplained syncope within the preceding 6 months [1]

6. In patients with HOCM who have spontaneous NSVT or an abnormal blood pressure response with exercise, with SCD high-risk features, if meaningful expected survival $>1$ year [1]

7. In patients with cardiac sarcoidosis and LVEF $\geq 35 \%$ with syncope and/ or evidence of myocardial scar by cardiac MRI or positron emission tomographic (PET) scan, and/or have an indication for permanent pacing, if meaningful expected survival $>1$ year [1]

8. In patients with cardiac sarcoidosis and LVEF $\geq 35 \%$, with inducible sustained VA, if meaningful expected survival $>1$ year [1]

9. In patients with cardiac sarcoidosis with indication for permanent pacing [1]

10. In patients with an LVAD and sustained VA[1]

11. In patients with Emery-Dreifuss and limb-girdle type IB muscular dystrophies with progressive cardiac involvement, if meaningful expected survival $>1$ year [1]

12. In pregnant patients needing ICD or VT ablation, it is reasonable to undergo these procedures after the first trimester of pregnancy [1]

13. For older patients with significant comorbidities, who meet indications for a primary prevention ICD, if meaningful expected survival >1 year [1]

14. In adults with repaired tetralogy of Fallot physiology and inducible VT/VF or spontaneous sustained VT, if meaningful expected survival >1 year[1]

15. In patients with repaired moderate or severe complexity adult congenital heart disease with unexplained syncope and at least moderate ventricular dysfunction or marked hypertrophy, if meaningful expected survival $>1$ year [1]

1. In patients with NICM, HF with NYHA class I and LVEF $\leq 35 \%$, despite GDMT, if meaningful expected survival $>1$ year [1]

2. In patients with HCM with NSVT or abnormal blood pressure response with exercise but without other SCD risk factors [1]

3. In patients with giant cell myocarditis with VF or hemodynamically unstable VT, if meaningful expected survival $>1$ year [1]

4. In patients with a heart transplant and severe allograft vasculopathy with LV dysfunction, if meaningful expected survival $>1$ year [1]

5. In patients with myotonic dystrophy type 1 with an indication for a permanent pacemaker, if meaningful expected survival > 1 year [1]

6. In asymptomatic patients with long QT syndrome (resting QTc $>500 \mathrm{~ms}$ ) while receiving a $\beta$-blocker [1]

7. In patients with adult congenital heart disease and severe ventricular dysfunction (LVEF < 35\%) and HF despite GDMT, if meaningful expected survival $>1$ year [1]

1. ICD is not indicated for NYHA class IV patients with medication-refractory $\mathrm{HF}$ who are not also candidates for cardiac transplantation, LVAD, or a CRT defibrillator[1]

2. In patients with incessant VT/VF, an ICD should not be implanted until sufficient control of the VA is achieved to prevent repeated ICD shocks [1]

3. In patients with medication-refractory NYHA class IV HF who are not also candidates for cardiac transplantation, LVAD, or a CRT defibrillator, ICD should not be implanted [1]

4. In patients with $\mathrm{HCM}$ genotype in the absence of SCD risk factors, ICD should not be implanted [1]

IHD - ischemic heart disease, VTIVF - ventricular tachycardia/ventricular fibrillation, LVEF - left ventricular ejection fraction, SCA sudden cardiac arrest, EPS - electrophysiological studies, GDMT - guideline-directed management and therapy, NYHA - New York Heart Association, HF- heart failure, NICM - non-ischemic cardiomyopathy, LVAD - left ventricular assist device, CRT - cardiac resynchronization therapy, HOCM - hypertrophic obstructive cardiomyopathy, NSVT - non-sustained ventricular tachycardia. 
Table II. Indications for WCD [1]

\begin{tabular}{|lc|}
\hline Indication [1] & Class [1] \\
\hline $\begin{array}{l}\text { In patients with ICD and a history of SCA or sustained VA in } \\
\text { whom removal of ICD is required (e.g. infection) [1] }\end{array}$ & $\begin{array}{c}\text { Class Ila } \\
\text { (The benefit outweighs the risk and it is } \\
\text { reasonable to administer the treatment) [1] }\end{array}$ \\
\hline $\begin{array}{l}\text { In patients at increased risk of SCD but not ineligible for ICD, such } \\
\text { as awaiting cardiac transplant, LVEF } \leq 35 \% \text { and are }<40 \text { days } \\
\text { post-MI, or have newly diagnosed NICM, revascularization within } \\
\text { the past } 90 \text { days, myocarditis or secondary cardiomyopathy [1] }\end{array}$ & $\begin{array}{c}\text { Class IIb } \\
\text { (The benefit probably outweighs the risk and } \\
\text { the treatment may be considered) [1] }\end{array}$ \\
$\begin{array}{l}\text { VA - ventricular arrhythmia, LVEF - left ventricular ejection fraction, SCA - sudden cardiac arrest, NICM - nonischemic cardiomyopathy, } \\
\text { MI - myocardial infarction. }\end{array}$
\end{tabular}

Table III. Indications for S-ICD [1]

\begin{tabular}{|lc|}
\hline Indication & Class \\
\hline $\begin{array}{l}\text { In patients meeting the criteria for ICD with inadequate vascular } \\
\text { access or at high risk for infection, and in whom pacing for bra- } \\
\text { dycardia or VT termination or as part of CRT is neither needed } \\
\text { nor anticipated [1] }\end{array}$ & $\begin{array}{c}\text { Class I } \\
\text { (The treatment is useful, its benefit greatly } \\
\text { outweighs the risk, and it should be } \\
\text { administered) [1] }\end{array}$ \\
\hline $\begin{array}{l}\text { In patients meeting the indication for ICD, if pacing for brady- } \\
\text { cardia or VT termination or as part of CRT is neither needed nor } \\
\text { anticipated [1] }\end{array}$ & $\begin{array}{c}\text { Class Ila } \\
\text { (The benefit outweighs the risk and it is } \\
\text { reasonable to administer the treatment) [1] }\end{array}$ \\
\hline $\begin{array}{l}\text { In patients meeting the indication for bradycardia pacing or CRT, } \\
\text { or for whom ATP for VT termination is required, S-ICD should not } \\
\text { be implanted [1] }\end{array}$ & $\begin{array}{c}\text { Class III } \\
\text { (The risk outweighs the benefit, and the } \\
\text { treatment should not be performed) [1] }\end{array}$ \\
\hline
\end{tabular}

$V T$ - ventricular tachycardia, CRT - cardiac resynchronization therapy, ATP - antitachycardia pacing.

system, for instance, pairs with the secure myMerlinPulse mobile app to help streamline communication and increase engagement between doctors and their patients. This sort of appbased ICD system which would allow patients to engage more frequently with their healthcare team by providing access to transmission history and device performance and schedule their appointments could be the immediate future of ICDs.

Application and refinements of global positioning systems (GPS) may allow remote monitoring and programming of pacemakers wherever patients are on the planet. Furthermore, these developments are ideal in rural and inaccessible areas. Incorporation of advanced technologies such as artificial intelligence (AI) in CRM devices will further help patients monitor their cardiac health by notifying them and their physicians in case of $a b$ normal activity. These cutting edge technologies come with cybersecurity threats and would therefore require the latest cybersecurity controls and partnering with industry cybersecurity experts to provide input into the design and testing of these controls to provide a seamless and a secure experience.

\section{Conclusions}

ICDs have come a long way since their first implantation in humans in 1980, firmly cementing their place in preventing SCD. Despite four decades of continual design improvements and upgrades, ICDs continue to evolve and current research is ongoing to refine the ever expanding indications for their use. With time, it is hoped that these devices will become more cost effective and safer.

\section{Conflict of interest}

The author declares no conflict of interest.

\section{References}

1. Al-Khatib SM, Stevenson WG, Ackerman MJ, et al. 2017 AHA/ACC/HRS Guideline for Management of Patients With Ventricular Arrhythmias and the Prevention of Sudden Cardiac Death: A Report of the American College of Cardiology/American Heart Association Task Force on Clinical Practice Guidelines and the Heart Rhythm Society. J Am Coll Cardiol 2017; 138: e272-391.

2. Welsenes GHV, Borleffs CJW, Rees JBV, et al. Improvements in 25 years of implantable cardioverter defibrillator therapy. Neth Heart J 2011; 19: 24. https://doi. org/10.1007/s12471-010-0047-3.

3. Coats W, Politte L, Flaker GC. The development and application of the implantable cardioverter defibrillator. Mo Med 2010; 107: 48-52.

4. Nisam S, Reddy S. The story of ... a lead. EP Europace 2015; 17: 677-88.

5. Beck C, Pritchard W, Feil S. Ventricular fibrillation of long duration abolished by electric shock. J Am Med Assoc 1947; 135: 985.

6. Kouwenhoven WB, Milnor WR, Knickerbocker GG, Chesnut WR. Closed chest defibrillation of the heart. Surgery 1957; 42: 550-61. 
7. Lown B, Amarasingham R, Neuman J. New method for terminating cardiac arrhythmias. Use of synchronized capacitor discharge. JAMA 1962; 182: 548-55.

8. Schuder JC, Stoeckle H, Gold JH, West JA, Keskar PY. Experimental ventricular defibrillation with an automatic and completely implanted system. Trans Am Soc Artif Intern Organs 1970; 16: 207-12.

9. Klein HU, Inama G. Implantable defibrillators: 30 years of history. G Ital Cardiol 2010; 11 (10 Suppl 1): 48S-52S

10. Mirowski M, Reid PR, Mower MM, et al. Termination of malignant ventricular arrhythmias with an implanted automatic defibrillator in human beings. $N$ Engl J Med 1980; 303: 322-4.

11. Winkle RA. Evolution of the implantable cardioverter-defibrillator: from bullets to BBs. J Am Coll Cardio 2012; 60: 2399-401.

12. Mirowski M, Mower MM, Staewen WS, et al. Standby automatic defibrillator. An approach to prevention of sudden coronary death. Arch Intern Med 1970; 126: 158-61.

13. Deyell MW, Tung S, Ignaszewski A. The implantable cardioverter-defibrillator: from Mirowski to its current use. BC Med J 2010; 52: 248.

14. Swerdlow CD, Friedman PA. Advanced ICD troubleshooting: part I. Pacing Clin Electrophysiol 2005; 28: 1322-46.

15. Kastor JA. Michel Mirowski and the automatic implantable defibrillator. Am J Cardiol 1989; 63: 1121-6.

16. Cannom DS, Prystowsky EN. The evolution of the implantable cardioverter defibrillator. Pacing Clin Electrophysiol 2004; 27: 419-31.

17. Bardy GH, Troutman C, Poole JE, et al. Clinical experience with a tiered-therapy, multiprogrammable antiarrhythmia device. Circulation 1992; 85: 1689-98.

18. Gradaus R, Breithardt G, Bocker D. ICD leads: design and chronic dysfunctions. Pacing Clin Electrophysiol 2003; 26: 649-57.

19. Boriani G, Ritter P, Biffi M, et al. Battery drain in daily practice and medium-term projections on longevity of cardioverter-defibrillators: an analysis from a remote monitoring database. Europace 2016; 18: 1366-73.

20. Wilcox JE, Fonarow GC, Zhang Y, et al. Clinical effectiveness of cardiac resynchronization and implantable cardioverter-defibrillator therapy in men and women with heart failure: findings from IMPROVE HF. Circ Heart Fail 2014; 7: 146-53.

21. Pokorney SD, Hellkamp AS, Yancy CW, et al. Primary prevention implantable cardioverter-defibrillators in older racial and ethnic minority patients. Circ Arrhythm Electrophysiol 2015; 8: 145-51.

22. Moss AJ. MADIT-I and MADIT-II. J Cardiovasc Electrophysiol 2003; 14 (9 Suppl): S96-8.

23. Bänsch D, Antz M, Boczor S, et al. Primary prevention of sudden cardiac death in idiopathic dilated cardiomyopathy: the Cardiomyopathy Trial (CAT). Circulation 2002; 105: $1453-8$.

24. Zareba W, Klein H, Cygankiewicz I, et al.; MADIT-CRT Investigators. Effectiveness of Cardiac Resynchronization Therapy by QRS Morphology in the Multicenter Automatic Defibrillator Implantation Trial-Cardiac Resynchronization Therapy (MADIT-CRT). Circulation 2011; 123: 1061-72

25. Gatzoulis KA, Tsiachris D, Arsenos P, et al. Arrhythmic risk stratification in post-myocardial infarction patients with preserved ejection fraction: the PRESERVE EF study. Eur Heart J 2019; 40: 2940-9.

26. Zaman S, Taylor AJ, Stiles M, Chow C, Kovoor P. Programmed Ventricular Stimulation to Risk Stratify for Early Cardioverter-Defibrillator Implantation to Prevent
Tachyarrhythmias following Acute Myocardial Infarction (PROTECT-ICD): trial protocol, background and significance. Heart Lung Circ 2016; 25: 1055-1062.

27. Auricchio A, Klein H, Geller CJ, Reek S, Heilman MS, Szymkiewicz SJ. Clinical efficacy of the wearable cardioverter-defibrillator in acutely terminating episodes of ventricular fibrillation. Am J Cardiol 1998; 81: 1253-6.

28. Feldman AM, Klein H, Tchou P, et al. Use of a wearable defibrillator in terminating tachyarrhythmias in patients at high risk for sudden death: results of the WEARIT/ BIROAD. Pacing Clin Electrophysiol 2004; 27: 4-9.

29. Dillon KA, Szymkiewicz SJ, Kaib TE. Evaluation of the effectiveness of a wearable cardioverter defibrillator detection algorithm. J Electrocardiol 2010; 43: 63-7.

30. Kleemann T, Becker T, Doenges $\mathrm{K}$, et al. Annual rate of transvenous defibrillation lead defects in implantable cardioverter-defibrillators over a period of 10 years. Circulation 2007; 115: 2474-80.

31. Bardy GH, Smith WM, Hood MA, et al. An entirely subcutaneous implantable cardioverter-defibrillator. N Engl J Med 2010; 363: 36-44.

32. Hauser RG. The subcutaneous implantable cardioverter-defibrillator: should patients want one? J Am Coll Cardiol 2013; 61: 20-2.

33. Auricchio A, Delnoy PP, Regoli F, Seifert M, Markou T, Butter C. First-in-man implantation of leadless ultrasound-based cardiac stimulation pacing system: novel endocardial left ventricular resynchronization therapy in heart failure patients. Europace 2013; 15: 1191-7.

34. Dillon JJ, DeSimone CV, Sapir Y, et al. Noninvasive potassium determination using a mathematically processed ECG: proof of concept for a novel 'blood-less, blood test'. J Electrocardiol 2015; 48: 12-8. 